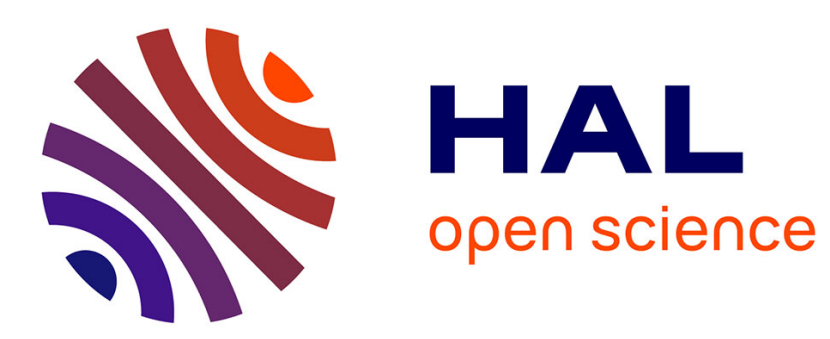

\title{
Genèse et résolution du désir oedipien
}

\author{
Girindrasekhar Bose, Thamy Ayouch
}

\section{To cite this version:}

Girindrasekhar Bose, Thamy Ayouch. Genèse et résolution du désir oedipien. Recherches en psychanalyse, 2005, 1, pp.189-203. 10.3917/rep.001.0189 . halshs-00940706

\section{HAL Id: halshs-00940706 https://shs.hal.science/halshs-00940706}

Submitted on 8 Feb 2014

HAL is a multi-disciplinary open access archive for the deposit and dissemination of scientific research documents, whether they are published or not. The documents may come from teaching and research institutions in France or abroad, or from public or private research centers.
L'archive ouverte pluridisciplinaire HAL, est destinée au dépôt et à la diffusion de documents scientifiques de niveau recherche, publiés ou non, émanant des établissements d'enseignement et de recherche français ou étrangers, des laboratoires publics ou privés. 


\section{GENESE ET RESOLUTION DU DESIR OEDIPIEN}

Girindrasekhar Bose

\section{Le complexe d'CEdipe}

L'une des premières découvertes de la psychanalyse fut celle du complexe d'CEdipe. Que l'inconscient pût receler des pensées incestueuses à l'endroit de la mère se révéla choquant pour plus d'un. Il semblait en effet difficile d'admettre qu'un esprit fût pervers au point de considérer de manière aussi dégradante le plus pur des rapports humains. Cette reconnaissance d'un fond sexuel à notre affection pour la mère n'a toutefois pas à provoquer une si grande surprise : la mythologie, le folklore et la littérature de tous les pays du monde abondent d'histoires sur de tels rapports incestueux. En outre, les plus purs et les plus nobles d'entre nous ont parfois d'explicites rêves incestueux. Les législateurs antiques convenaient que la loi dût inclure des mesures de punition de l'inceste. Dans les Lois de Manu ${ }^{l}$ est clairement formulée l'interdiction pour un homme de partager le siège de sa mère, de sa fille ou de sa sœur en l'absence d'une tierce personne, car, dit-on, les passions et les appétits sont bien fougueux et risquent d'ébranler même les plus doctes. L'histoire la plus célèbre d'inceste maternel comparable aux singularités de l'inconscient est celle de l'Oedipus Tyrannus de Sophocle. Le désir inconscient d'inceste s'est vu attribuer le nom de complexe d'Edipe du fait de l'analogie qu'il offre avec la situation d'CEdipe dans la tragédie.

Laios, roi de Thèbes, demanda à Apollon de Delphes de lui accorder un enfant. Sa prière fut exaucée, mais on lui fit entendre que son fils le tuerait, réalisant ainsi la malédiction de Pelops dont il avait kidnappé l'enfant. Jocaste, épouse du roi Laios, donna naissance à un garçon. Pour échapper à son destin, Laios, perça et attacha les pieds de l'enfant et ordonna à son serviteur d'abandonner le nourrisson dans le désert. Le serviteur confia l'enfant à un berger qui le remit au roi Polybe. Celui-ci le nomma CEdipe, ce qui signifie pieds gonflés, et l'adopta. Devenu adulte et curieux de connaitre son origine, Edipe consulta l'oracle qui lui indiqua qu'il tuerait son père et épouserait sa mère. Pour éviter qu'un tel destin ne s'accomplît, Edipe quitta son lieu d'adoption, mais il rencontra par hasard Laios, son propre père, et le tua lors d'une rixe. Il perça l'énigme du Sphinx, fut, en récompense, déclaré roi de Thèbes et reçut pour épouse sa propre mère endeuillée, Jocaste. La peste ravagea alors Thèbes dont les habitants consultèrent l'oracle, qui leur affirma que le fléau cesserait si CEdipe était chassé du pays. Devenu conscient de ses véritables liens avec Jocaste et Laios, Edipe s'aveugla luimême, par repentir, et se condamna à l'exil.

Si dans l'histoire CEdipe tua son père, c'est une même hostilité contre le père qui se manifeste dans l'inconscient, et qu'accompagne l'amour de la mère, traits conjoints du complexe d'Edipe. Par delà cette hostilité à l'endroit du père, la configuration oedipienne révèle invariablement d'autres traits qui n'ont pas suffisamment été étudiés par les psychanalystes. Dans cet article, je tenterai de montrer la grande complexité de cette situation, et de souligner les nombreux aspects qui s'en révèlent indissociables. Certains de ces aspects se manifestent parfois de manière patente en analyse et peuvent retenir notre attention. Outre donc ces

\footnotetext{
${ }^{1}$ Manu est un héros mythique qui serait l'auteur du Manava-Dharma-Shâstra, communément appelé "Lois de Manu". Ce traité religieux, moral et social expose les lois du Dharma, ou ordre du monde, dont la division de la société en quatre varna et en milliers de jâti - ce que nous appelons les castes - fait partie (Note de Jean Nimylowycz)
} 
repères éminents dans la configuration œdipienne, un observateur minutieux pourra toujours relever d'autres traits moins saillants et néanmoins d'une importance égale dans le tissage de la trame œdipienne. Les liens entre ces différents éléments se révèlent progressivement, à mesure qu'augmente le nombre de cas analysés. La théorie de la genèse du complexe d'Edipe ici présentée prend appui sur des analyses réelles. Mes conceptions diffèrent quelque peu, et sur certains points, de celles déjà développées sur ce sujet. J'ai très librement cité Freud pour exposer la manière dont la plupart des chercheurs considèrent le problème. Le rappel des positions freudiennes effectué ici permettra de souligner les différences propres à mon point de vue.

\section{La sexualité infantile}

Dans ses Trois essais sur la théorie sexuelle, Freud mit en exergue certaines singularités du développement de la libido infantile. Selon lui, le choix d'objet sexuel s'effectue dès la petite enfance, de telle façon que toutes les motions sexuelles se dirigent vers une seule personne dans laquelle elles cherchent à trouver leur satisfaction. La libido infantile ressemble alors étroitement à celle de l'adulte. Pour seule différence, Freud releva la distribution plus ou moins uniforme de la libido, pendant l'enfance, sur les diverses zones érogènes. Les organes génitaux ne reçoivent alors aucune signification sexuelle spéciale, le primat des parties génitales n'étant pas réalisé. Des découvertes plus tardives engagèrent Freud à changer sa conception. Dans un article intitulé «L'organisation génitale infantile » paru dans la livraison d'avril 1924 de l'International Journal of Psychoanalysis, Freud soutient :

« Je ne serais plus satisfait actuellement de la proposition selon laquelle le primat des organes génitaux ne s'effectuerait dans la première enfance que d'une façon très imparfaite ou pas du tout. La vie sexuelle de l'enfant se rapproche de celle de l'adulte dans une bien plus grande mesure et cela ne concerne pas seulement la survenue d'un choix d'objet. Même si on ne parvient pas à une véritable synthèse des pulsions partielles sous le primat des organes génitaux, au faîte du développement de la sexualité infantile, l'intérêt pour les organes génitaux et l'activité génitale acquièrent cependant une importance dominante qui ne le cède que peu à celle de la maturité. Le caractère principal de cette "organisation génitale infantile » est en même temps ce qui la différencie de l'organisation génitale définitive de l'adulte. Il réside en ceci que pour les deux sexes, un seul organe génital, l'organe mâle, joue un rôle. Il n'existe donc pas un primat génital mais un primat du phallus $»^{2}$.

Freud poursuit : «Malheureusement, nous ne pouvons décrire cet état de choses que chez l'enfant mâle; la connaissance des processus correspondants chez la petite fille nous fait défaut $»^{3}$. Le petit garçon commence par croire que la fille, comme lui, est pourvue d'un pénis. La vue fortuite des organes génitaux d'une petite fille fournit d'abord au garçon l'information que la fille est constituée différemment. Il s'efforce de croire nonobstant qu'elle a un pénis, qu'il est petit, qu'il grandira, etc. S'il a fait l'objet, à cette période, de réprimandes et de menaces de castration visant les attouchements inconvenants de son pénis, il considère alors l'absence de celui-ci chez la petite fille comme un résultat de la castration, et ses propres peurs ici éveillées donnent lieu au développement de ce qui est appelé complexe de castration. La menace de castration prend tout son sens uniquement lors du primat du stade génital. L'absence de pénis chez les filles est cause de dépréciation sexuelle aux yeux du petit garçon et représente l'un des facteurs de la genèse de l'homosexualité. Puisque la castration ne peut

\footnotetext{
${ }^{2}$ «L'organisation génitale infantile», trad. J. Laplanche, in La vie sexuelle, PUF, Paris, 1969, p 114

${ }^{3}$ ibid.
} 
advenir que comme châtiment, le petit garçon en vient à penser que seules des personnes féminines indignes perdent leur pénis, mais que des femmes respectées, comme la mère, le gardent intact. Plus tard, lorsque le garçon apprend que seules les femmes peuvent porter un enfant, la mère est dessaisie du pénis, en échange duquel un enfant lui est accordé. C'est bien ultérieurement qu'est acquise la connaissance du véritable état des choses ${ }^{4}$.

\section{La conception freudienne de la disparition du complexe d'CEdipe}

Dans un article intitulé « La disparition du complexe d'Edipe » paru dans le numéro du mois d'octobre 1924 de l' International Journal of Psychoanalysis, Freud a exposé les facteurs qui entraînent la disparition du complexe d'Édipe. Celui-ci forme le phénomène central de la période sexuelle de la première enfance, mais le refoulement un fois installé en précipite la disparition. Suit alors ce que l'on connaît sous le nom de période de latence. Deux théories sont avancées pour rendre compte de ce refoulement. L'une soutient qu'il est provoqué par la douloureuse déception et l'absence de satisfaction des désirs qui forment le complexe d'Edipe; l'autre qu'il s'agit d'un déclin prédéterminé dans le cours naturel du développement mental de l'enfant. Ces deux conceptions ne sont pas incompatibles, mais il semble nécessaire de chercher le mécanisme exact par lequel ce déclin est provoqué.

La phase phallique est contemporaine du complexe d'Edipe ; elle ne mène pas directement au développement adulte, mais est engloutie par le temps de latence. Selon Freud, la phase phallique de l'organisation génitale succombe à la menace de la castration sous l'influence d'autres facteurs. L'enfant a été préparé à la perte de parties corporelles précieuses, lors de l'expérience du retrait du sein et de l'évacuation du contenu de l'intestin. Le coup de grâce est donné lorsque l'enfant voit l'organe génital féminin, parfois fortuitement chez une petite fille. La perte de son propre pénis devient alors dangereusement possible, et la menace de castration prend tout son effet. Le complexe d'Edipe offre à l'enfant deux possibilités de satisfaction, l'une active et l'autre passive. Il peut se mettre à la place de son père et avoir commerce avec la mère, ou inversement remplacer la mère. Là, le père devient un rival, fort susceptible alors d'exercer la castration ; ici, jouer le rôle de la femme exige comme condition préalable l'abandon du pénis. Dans les deux cas, le petit garçon en vient donc à perdre son précieux organe. Un conflit apparaît alors entre l'intérêt narcissique pour le pénis et l'investissement libidinal des objets parentaux. Par un processus d'identification avec les parents, l'autorité de ces derniers est introjectée dans le moi de l'enfant et forme le noyau du surmoi qui, en imposant, à la manière du père, des restrictions, tient les tendances œdipiennes en échec. Les motions sexuelles œdipiennes sont alors transformées et sublimées pour faire place à de l'affection. Ce procès introduit le temps de latence, et a pour résultat la destruction et la suppression du complexe d'Edipe lors des dénouements les plus heureux. Il s'agit alors d'un procès qui dépasse le refoulement. Lorsque le résultat n'est pas aussi abouti, le complexe d'Edipe persiste inconsciemment, et se manifestera plus tard dans quelque effet pathogène.

Notre connaissance du complexe d'CEdipe chez le sexe féminin est assez élémentaire. Selon Freud, le sexe féminin lui aussi développe un complexe d'CEdipe, un surmoi, et un temps de latence, et connait, lui aussi une organisation phallique et un complexe de castration, toutefois de manière différente. La petite fille croit en l'existence du phallus chez la femme adulte, et pense qu'elle obtiendra en grandissant un appendice aussi important. Lorsqu'elle prend

\footnotetext{
${ }^{4}$ G. Bose cite « librement » l'article mentionné de Freud, en en reprenant quelques phrases qu'il réinsère dans les siennes propres. Nous conservons ici de la traduction de J. Laplanche certains choix et expressions, tels « personnes féminines indignes » ou « la mère est dessaisie du pénis » $(\mathrm{NdT})$
} 
connaissance de la menace de castration, elle croit que son propre phallus a été coupé. Puisque la castration est un fait accompli dans son cas, la crainte qu'elle pourrait provoquer reste sans effet. Le motif de formation du surmoi et de démolition de l'organisation sexuelle infantile en est alors affaibli. Dans son cas, c'est l'influence de l'éducation et la menace de perte de l'amour qui entraînent la modification de la sexualité. Freud est d'avis que le complexe d'Edipe chez les filles est plus simple que chez les garçons, et s'étend rarement audelà du désir de remplacer la mère. Trouvant dans l'enfant une compensation de la perte du pénis, la fille s'attend à recevoir un enfant de son père, vœu condamné à n'être jamais exaucé. La faible dimension sadique de la libido chez la fille, en comparaison avec le garçon, à attribuer, selon Freud, à l'absence de pénis, facilite la sublimation des tendances sexuelles et leur transformation en motions tendres.

Freud écrit :

«Je ne doute pas que les relations temporelles et causales que nous décrivons ici entre complexe d'CEdipe, intimidation sexuelle ( menace de castration), formation du surmoi et entrée en scène du temps de latence soient d'une espèce typique ; mais je ne veux pas affirmer que ce type est le seul possible. Des changements dans la suite temporelle et dans l'enchaînement de ces processus devront être très lourds de signification pour le développement de l'individu.

Depuis la publication de l'intéressante étude d'Otto Rank sur Le traumatisme de la naissance, on ne peut même pas accepter sans autre discussion le résultat de cette petite recherche, à savoir que le complexe d'Edipe du garçon périt du fait de l'angoisse de castration. Mais il me semble prématuré d'entrer maintenant dans cette discussion $»^{5}$.

\section{Riposte et identité}

Dans le cadre de ma théorie des désirs opposés, j'ai montré dans d'autres développements que lorsqu'un individu est frappé, il ressent l'envie de frapper l'agresseur en retour, et si cette envie n'a pas l'occasion d'être satisfaite, une douloureuse tension s'ensuit. Le désir de riposte ou de vengeance suit la loi du talion - œil pour œil, dent pour dent. Conformément à ma théorie des désirs opposés, la satisfaction, volontaire ou non, du moindre désir s'accompagne systématiquement du développement du désir opposé. Tout individu éprouve l'envie inconsciente d'être frappé : si celle-ci vient à être satisfaite involontairement lors d'une agression, son opposé, l'envie de frapper, apparaît consciemment, comme désir de vengeance. Lorsque des circonstances particulières favorisent la satisfaction répétée d'une seule sorte de désir, l'envie consciente de se comporter de manière opposée se développe progressivement et finit par se libérer dans une action. Si les occasions de satisfaire le désir opposé viennent à manquer, la tension s'accumule dans le système. Parfois, le désir opposé ne peut pas recevoir d'assentiment social, car la riposte n'est pas toujours une possibilité réalisable dans une société civilisée. Les tensions sont alors soulagées par des actions symboliques, des rêves éveillés, ou tout autre moyen permettant la satisfaction imaginaire du désir. Si l'individu a eu antérieurement l'occasion d'assouvir des tendances similaires ou identiques au désir interdit, une solution bien plus satisfaisante et pérenne sera alors apportée à la tension. Dans ce cas là apparaît même parfois une identification avec l'agent à l'origine de la provocation : le sujet se place dans la position de l'agresseur et peut alors comprendre ${ }^{6}$

\footnotetext{
5 « La disparition du complexe d'CEdipe », trad. D. Berger, in La vie sexuelle, PUF, Paris, 1969, p 122

${ }^{6}$ Il s'agit ici du verbe « sympathise », dont Bose reprend le substantif, " sympathy » dans les parties suivantes. Ces vocables seront tour à tour traduits par « comprendre », " parfaite intelligence », et « compréhension » $(\mathrm{NdT})$
} 
ses motifs et ses actions. Le pardon, l'indulgence et la résignation sont ainsi possibles, et toute la tension s'en trouve soulagée. En effet, lorsque le désir de vengeance est assouvi, l'identification a lieu plus facilement. La vengeance ou la riposte permettent seulement l'identité d'action avec l'agresseur, et représentent une étape préliminaire vers l'identification avec le sujet. La riposte consiste à renverser la relation sujet-objet, l'action demeurant invariée. L'identification, elle, déplace le moi vers la position de l'objet. L'identité entraîne l'expansion du moi. Une personne identifiée avec une autre s'intéresse à ses différentes activités. Pour des raisons de commodité, j'appellerai le mécanisme de riposte «identité d'action » et la véritable identification « identité du moi ».

\section{Le triangle œdipien}

L'enfant reçoit un certain nombre d'attentions de la mère qui rendent possible la satisfaction d'un type d'appétence ${ }^{7}$ particulier que je nommerai appétence enfant-mère. Les désir d'être nourri, caressé par la mère, etc. appartiennent à cette catégorie. La satisfaction continue et répétée de ces appétences donne lieu à l'émergence de la catégorie d'envies opposées, nommément les appétences mère-enfant, désirs de nourrir un enfant, de le caresser etc. Cela mène, initialement, à l'identité d'action uniquement, et l'enfant caresse sa mère en échange de ses soins, feint de la nourrir, de la coucher, et de se livrer à de tels jeux. Lorsque la voie de l'identification a été largement ouverte par de telles actions, l'enfant entreprend de se mettre dans la position de la mère (identité du moi) et trouve du plaisir à jouer à la maman avec ses poupées. Cela reste vrai pour les garçons comme pour les filles.

L'identification du moi de l'enfant avec la mère conduit à une extension de son $\operatorname{soi}^{8}$. Il commence à s'intéresser à ce pour quoi la mère montre de l'intérêt; le père, devenu alors un nouveau pôle d'attention, est appréhendé depuis la perspective de la mère. Si le père est aussi affectueux que la mère, les relations enfant-père et père-enfant connaissent le même développement que celles entretenues avec la mère, et l'enfant entreprend d'imiter les activités du père. L'identification commençante avec la mère entraîne chez l'enfant le développement, d'une nouvelle approche et d'une nouvelle signification des relations enfantpère père-enfant. L'enfant perçoit le père du point de vue de la mère : il se comporte comme la femme de son père. Cette phase a pour ultime stade de développement l'identification avec le père via la mère ; l'enfant endosse alors le rôle de son père et considère sa mère comme son épouse.

Identité d'action et identité du moi permettent à l'enfant d'appréhender la relation triangulaire enfant-mère-père à partir de trois angles différents. Depuis son propre point de vue, il expérimente les sentiments d'un enfant pour sa mère et son père, et leurs différents sentiments réciproques à son endroit. Du point de vue de sa mère, il prend connaissance des sentiments d'une femme pour son enfant et son mari, et des sentiments réciproques. Du point de vue du père, il éprouve les sentiments d'un homme pour son enfant et sa femme, et les sentiments réciproques. Toute cette situation pourrait se voir figurer sous forme d'un schéma.

Si l'identification a lieu sans le moindre problème, cela donne lieu à une parfaite entente et une parfaite intelligence ${ }^{9}$ avec les parents, ce qui est rarement le cas. Bien des facteurs peuvent empêcher le mécanisme d'identification de s'opérer sans heurts. En présence

\footnotetext{
${ }^{7}$ Il s'agit ici du terme anglais « craving », désir ardent, pour lequel cette traduction a été choisie de manière à le distinguer de «wish» - traduit ici le plus souvent par désir - et « desire » - que rendent tour à tour « désir » et « envie » $(\mathrm{NdT})$

${ }^{8}$ « Moi » et « soi » renvoient ici aux termes anglais « ego » et « self» $(\mathrm{NdT})$

${ }^{9}$ Cf. note 5
} 
d'influences unilatérales, le refoulement et ses conséquences entrent en jeu. La sexualité grandissante de l'enfant investit la triple relation d'un sens sexuel qui s'avère d'une importance insigne pour la vie de l'individu. La coloration sexuelle de cette triple relation apparaît très tôt. Si l'on garde à l'esprit que la sexualité infantile, avant l'avènement du temps de latence, présente une grande ressemblance avec sa forme adulte, il semble alors peu surprenant que des images sexuelles particulièrement complexes ainsi que toutes les situations sexuelles possibles traversent l'esprit de l'enfant. Du point de vue de la mère, l'enfant éprouve le désir d'être la femme de son père et d'en porter la progéniture. Son caractère acquiert ainsi une féminité qui se manifeste également dans son commerce avec d'autres personnes. Du point de vue du père, l'enfant se comporte en homme dominateur, veut engendrer un enfant avec sa mère, etc.

Ces différents points de vue suscitent, comme répercussions secondes, des formations dont l'importance ne saurait être surestimée. L'attitude féminine envers le père donne lieu au développement d'une homosexualité passive ainsi qu'à d'autres phénomènes seconds. Notons en outre que certaines de ces attitudes s'excluent mutuellement. Les rôles de mère et de père, par exemple, ne peuvent advenir à la conscience simultanément. Dans des conditions normales, la satisfaction d'une attitude entraîne celle de l'attitude opposée, selon le mécanisme d'identité inconsciente, comme je l'ai démontré dans d'autres développements. Pour être satisfaite, la position féminine requiert une pré-conception des caractéristiques masculines, ce qui implique une identification inconsciente avec l'homme, et vice-versa. Comme il a été souligné précédemment, si ces procès sont unilatéraux, le refoulement a lieu, et le mécanisme d'identification est mis en échec, ce qui donne lieu à un conflit interne entre les types opposés de désirs. C'est seulement dans de telles conditions que le complexe d'CEdipe fait son apparition. Lorsque l'identification a lieu sans heurts, il y a parfaite intelligence des trois points de vue. Je prétends que le complexe d'EEdipe ne peut être résolu que dans de telles circonstances favorables. Tout le long d'une cure psychanalytique, cette lutte entre des tendances contradictoires peut être observée, et s'accompagne de la prévalence sur le plan conscient tantôt de l'une, tantôt de l'autre. Lorsque l'une des attitudes l'emporte, l'attitude opposée est très peu tolérée. A mesure que se met en place la résolution, l'identification avec l'attitude opposée est de plus en plus réelle, entraînant finalement une compréhension des deux points de vue opposés.

Je vais maintenant indiquer le déroulement d'un tel conflit, et les formations qui en résultent comme répercussions secondes.

\section{Les répercussions odipiennes}

Commençons par analyser ici l'attitude de l'enfant avec sa mère. Lorsque le moi de l'enfant entreprend de se différencier du monde environnant, les expériences passives ont un rôle principal. A mesure que l'enfant se développe, il découvre progressivement que la plupart de ses impressions majeures lui viennent de la mère. La soumission répétée à de telles situations de passivité suscite, chez l'enfant, des désirs actifs de la même nature, selon ce que j'ai précédemment décrit sous le nom de mécanisme d' "identité de réaction ». L'enfant agit à l'instar de la mère, en la prenant elle-même pour objet : si ses caresses ont été passionnées, l'enfant réagira de la même façon en y ajoutant la coloration sexuelle résultant du développement des tendances sexuelles qu'il connaît alors. Singulièrement, cette première phase d'attachement à la mère est rarement exhumée lors d'une analyse. Elle ne semble pas être une composante importante du complexe d'Edipe. Si dans certains cas, cette phase sexuelle primaire s'avère prépondérante, il y a alors de fortes chances que la haine œdipienne typique à l'endroit du père soit absente, puisque ce dernier ne semble pas entrer du tout en jeu. 
L'identité d'action fait progressivement place à l'identité du moi, et l'enfant se place dans la position de la mère. Il joue avec ses poupées qu'il imagine être ses enfants, considère son père comme un mari, et, s'il a l'occasion d'être par hasard témoin des ébats parentaux, adopte imaginairement, lors de cette phase, la position passive de la mère. Une véritable attitude sexuelle féminine vis à vis du père émerge, et peut même culminer dans le désir d'en obtenir un enfant. A ce stade du développement sexuel, les organes génitaux de l'enfant éprouvent déjà de riches sensations, ce qui, accompagné de la connaissance qu'il gagne des différences dans l'organisation sexuelle masculine et féminine, pousse le garçon à souhaiter le retrait de son phallus. Il n'est pas inutile de préciser ici que les enfants indiens acquièrent très tôt une véritable connaissance des organes génitaux des deux sexes.

Le désir de castration est ainsi une conséquence naturelle de l'envie d'être une femme. L'enfant, pour s'amuser, demande parfois à sa mère de le déguiser en femme et joue le rôle d'épouse avec d'autres enfants ou même avec des adultes. Ce jeu, communément appelé « bow bow khela » ou « jeu de l'épouse » est, dans ce pays, particulièrement répandu chez les filles comme chez les garçons d'un certain âge. Je me rappelle le cas singulier d'un jeune garçon qui s'habillait toujours en fille et, pour parfaire la ressemblance, attachait un morceau de fil autour de son prépuce pour ramener le pénis tout contre le scrotum en fixant l'autre extrémité du fil dans son dos, comme un bandage autour de la taille. Le pénis était ainsi recouvert par les plis du scrotum, et l'appareil génital entier ressemblait à s'y méprendre à un organe féminin.

L'envie d'être une femme, ou sa modification, le désir de castration, peut être régulièrement identifié dans toutes les analyses. L'angoisse de castration n'est qu'une formation tardive, qui fonctionne comme défense devant ce désir. Comme je l'ai mentionné dans mon article concernant la genèse de l'homosexualité, la menace de castration est particulièrement commune dans la société indienne, mais le complexe de castration tel que le décrivent des observateurs européens est rarement repérable chez des patients indiens. Dans mes cas européens, le complexe de castration est souvent apparu durant l'analyse, mais il a, ici aussi, presque toujours été relié à l'envie d'être une femme. J'indiquerai ultérieurement comment la menace de castration provoque le développement du complexe de castration.

Chez la petite fille, toute la constitution psycho-sexuelle favorise l'attitude féminine envers le père, si bien que cette phase est beaucoup plus développée que chez le garçon. Comme je l'ai indiqué ailleurs, cela s'avère déterminant quant à la formation de l'hystérie.

Chez le garçon, hormis la castration, une autre possibilité d'endosser un rôle féminin se présente. L'anus, zone érogène également importante pour l'enfant, peut remplacer l'organe génital féminin ; l'enfant développe alors, dans la phase sexuelle féminine, une homosexualité passive en relation avec le père. Lors de cette phase, les fèces sont un symbole de l'enfant, ce que les théories infantiles de la naissance par l'anus ne manquent pas de conforter. Dans cette position féminine ou homosexuelle passive, l'enfant imagine de possibles configurations sexuelles avec le père dont il interprète le comportement à la lumière de sa propre attitude. Le recours imaginaire continu à ces désirs passifs suscite l'apparition des désirs actifs correspondants, par le mécanisme d'identité d'action, et l'enfant assume alors un rôle masculin actif vis à vis du père, qu'il place imaginairement dans la position d'une femme ou d'un homosexuel passif. Il peut même en venir à se représenter son père dépourvu de pénis, et donnant naissance à un enfant. La phase d'identité d'action laisse progressivement place à celle d'identité du moi, et le moi de l'enfant s'éprouve comme celui du père. Les intérêts du père deviennent ceux de l'enfant, qui se figure alors être un homme adulte comme son père. La mère, nouvel objet sexuel, est appréhendée du point du vue du père. J'ai appelé cette phase 
« le point nodal œdipien ${ }^{10}$ dans mon article sur la genèse de l'homosexualité. L'enfant souhaite féconder la mère et lui donner un enfant. Il prend également connaissance de l'attitude du père envers son fils et joue un rôle de protecteur avec ses plus jeunes frères, avec d'autres enfants et aussi avec la mère. Ainsi l'enfant mesure-t-il les implications du triple rôle d'enfant, de mère et de père. Si le développement suit le cours que j'ai décrit plus haut, l'enfant ne nourrit pas de haine à l'endroit du père, et l'intelligence ${ }^{11}$ avec les trois personnes $\mathrm{du}$ trigone enfant-mère-père est totale. Les tendances sexuelles envers les parents sont rapidement sublimées et transformées en affection. Il n'y a pas de fixation parentale de la libido, et les possibilités d'un développement sexuel normal sont alors intactes. Pour que cet état des choses idéal puisse avoir lieu, il est nécessaire que le comportement des parents permette à l'enfant de satisfaire, au moins en imagination, ses tendances opposées actives et passives les concernant. Un savant équilibre de concessions et d'exigences est pour les parents la condition requise. Malheureusement, l'enfant est très souvent soumis à des influences unilatérales seulement, ce qui provoque l'avènement du refoulement à une phase ou une autre du développement sexuel, et l'émergence du complexe d'CEdipe avec ses inhibitions complexes. Les analyses de cas œdipiens ne manquent jamais de révéler les différentes phases de la triple relation que j'ai précédemment soulignées, et leur résolution dans la cure n'a lieu que lorsque advient l'intelligence de chacun des trois acteurs dans cette relation triangulaire.

\section{Le désir oedipien}

Je vais maintenant exposer le mode de surgissement de la perturbation œdipienne, puis je décrirai les différentes manifestations qui s'y rapportent. Notons toutefois qu'à une manifestation pathologique particulière correspond souvent une surdétermination: sa formation peut être provoquée par plusieurs facteurs différents. Je tire la plus grande partie de mon information clinique d'analyses de sujets adultes masculins et féminins. Je précise toutefois que ces découvertes se sont complètement confirmées dans quelques cas d'enfants que j'ai eu l'occasion d'analyser.

Comme je l'ai précédemment dit, la phase primaire des relations enfant-mère mère-enfant apparaît rarement dans l'analyse de cas œdipiens ; je ne peux donc identifier en toute certitude aucune perturbation issue de ce stade du développement. Les phases suivantes, en revanche, apparaissent régulièrement en analyse. Les possibilités de mauvaise résolution de l'attitude féminine de l'enfant envers son père sont frappantes. Si la libido se fixe dans cette attitude, la femme est incapable d'aimer un autre homme que son père. Parfois, le choix de l'objet d'amour est déterminé par quelques caractéristiques du père présentes chez celui qu'elle épouse. Les manifestations hystériques peuvent être rapportées à ce facteur.

Chez le garçon, la fixation de la libido dans l'attitude féminine est responsable du développement d'aspects féminins et d'une homosexualité passive. Une timidité anormale en présence de ses supérieurs et un défaut d'affirmation de soi deviennent alors des traits marqués. Sont également observables certaines maladies ainsi que des symptômes névrotiques représentant le coït du point de vue féminin et l'accouchement, dans lesquels l'image du père ou un de ses substituts assurent le rôle actif. Des pensées de castration, des rêves de menstruation et l'impuissance sexuelle sont autant de fréquentes manifestations. Le sujet

\footnotetext{
${ }^{10} \mathrm{C}$ 'est ici la traduction du concept de « Oedipus point » de Bose que propose Jean Nimylowycz dans son travail sur cet auteur (NdT)

${ }^{11}$ Cf. note 5
} 
s'adonne à des rêveries éveillées figurant une relation sexuelle dans laquelle il s'identifie au partenaire féminin.

A cause du refoulement, un conflit a toujours lieu, parfois même dans la satisfaction imaginaire de ces désirs féminins; le sujet tentera donc de mettre en place une forme de défense. Plusieurs symptômes abdominaux perturbant l'évacuation normale de l'intestin peuvent alors apparaître. Les fantasmes d'agression sexuelle par le père, personne révérée, sont souvent modifiés par l'introduction d'hommes de basse extraction et de voyous pour jouer le rôle de l'agresseur sexuel. Lorsque la défense se consolide, les désirs passifs font place à de la peur. Celle-ci se manifeste alors, avec une intensité obsessionnelle, comme crainte d'une agression - se révélant de nature sexuelle après analyse -, ou peur de voleurs représentant autant d'images du père. Le désir de castration se transforme alors en angoisse de castration, dans laquelle le père ou l'un de ses substituts est l'agresseur. La menace de castration ne devient effective que lors de cette phase. Le complexe du petit pénis peut $s$ 'avérer en partie déterminant dans cette attitude féminine.

L'identité d'action marque l'étape suivante de la défense. Rappelons ici que ce mécanisme est un moment préliminaire vers celui d'identité du moi, entraînant la compréhension ${ }^{12}$ de l'auteur de l'agression. La défense mise en place par ce mécanisme d'identité du moi est alors en même temps un phénomène favorisant la levée du refoulement. Dans la phase d'identité d'action, l'enfant tente de faire du père une femme envers laquelle il assume le rôle masculin agressif. Dans l'imagination de l'enfant, le père est traitée comme une femme, a un enfant ou est mis en position d'homosexuel passif. La composante sadique de la libido trouve alors une voie de décharge, et une attitude hostile à l'endroit du père se développe. La menace de castration vise maintenant le père, comme riposte de l'enfant. L'hostilité œdipienne à l'endroit du père peut être directement rapportée à cette phase du développement de l'enfant. Elle n'est pas alors animée de la seule rivalité pour la possession de la mère, mais présente une coloration sexuelle distincte. L'homosexualité active qui visait d'abord le père peut aussi être dirigée vers d'autres objets d'amour du même âge ou plus jeunes. La rôle féminin du père est doté d'une signification particulièrement intéressante : lorsque s'établit l'identité du moi avec le père, phase suivante du développement, l'amour œdipien pour la mère apparaît, et la position féminine du père est transférée sur la mère. La mère œdipienne est donc une image hétéroclite : elle représente le père et la mère.

Si la libido reste fixée à la phase d'identité d'action de la relation père-mère, l'enfant masculin devient homosexuel. Pour la fille, l'homosexualité se développe si la libido reste fixée au point nodal œdipien. La première phase de l'homosexualité pour la fille est de type actif, alors qu'elle est passive pour le garçon. J'ai abordé cela avec plus de précision dans mon article sur la genèse de l'homosexualité. La jalousie ressentie envers le partenaire sexuel peut être rapportée à l'homosexualité, et à la féminité chez le garçon ou à la masculinité chez la fille.

Le point nodal œdipien est véritablement initié lorsque s'enclenche l'identité du moi avec le père, et que le rôle d'objet sexuel échoit à la mère. Surgit alors le désir de lui donner un enfant. Les anomalies de développement lors de cette phase se traduisent chez le garçon par le choix de femmes âgées comme objets d'amour. La respectabilité de l'image de la mère est contournée par la substitution à la mère de la sœur, ou de femmes de basse extraction, ou même de prostituées. La puissance sexuelle est diminuée, et le sujet éprouve la peur obsessionnelle de ne pas parvenir à satisfaire une femme lors d'un rapport sexuel. Le complexe du petit pénis en est alors renforcé, le pénis de l'enfant étant considéré trop petit pour la mère. L'identité d'action dans la configuration père-mère entraîne l'adoption d'une

\footnotetext{
${ }^{12}$ Cf. note 5
} 
attitude passive dans le coït, le sujet préférant que la partenaire assume le rôle actif. Avec l'avènement de l'identité du moi dans la relation père-mère, la femme est comprise ${ }^{13}$ depuis le point de vue de l'homme, et le complexe d'Edipe est résolu. Ce n'est donc pas à la menace de castration que le désir oedipien succombe, mais à la faculté de mettre le moi dans la position de la mère dans la relation mère-père, et à celle de défier le père et de le castrer, c'est-à-dire de l'appréhender comme une femme. Dans l'histoire d'Edipe, le héros trouve un dénouement à son infortune en renonçant à son objet d'amour, et en s'aveuglant, en effectuant donc une castration symbolique. Il jette les armes devant l'image paternelle qui persiste bien qu'il ait tué le père, et se transforme en femme devant lui. La solution est loin d'être idéale, et semble bien peu réalisable dans la société actuelle.

La configuration œdipienne chez la fille présente certaines singularités dont l'étude est instructive. Chez les femmes indiennes, le désir d'avoir un garçon plutôt qu'une fille est presque universel. Le contexte social favorise sans nul doute cette attitude; il semblerait toutefois que prévale également une forte détermination inconsciente. Si dans la relation pèremère, le désir naturel d'un enfant serait d'avoir une progéniture du même sexe que lui, pourquoi alors les filles développent-elles une préférence pour un enfant masculin ? La raison semble résider dans l'attitude de certaines femmes normales qui ne veulent pas seulement un garçon, mais un garçon à l'image du père. Le fils est alors une image composite de l'enfant et de son père. C'est pourquoi il semble naturel de supposer une coloration sexuelle à l'amour d'une mère pour son enfant. Bien des femmes pourraient reconnaître avoir éprouvé un type de plaisir particulier en donnant le sein à leur nourrisson. Chez certaines névrosées, cet élément se révèle distinctement en analyse. Une patiente âgée souffrant de psychonévrose obsessionnelle développa un fort transfert positif de type clairement sexuel. Son attitude envers le médecin était tantôt celle d'une mère avec son fils, et tantôt celle d'une fille avec son père. Le médecin incarnait l'image composite du fils, du père et du mari. Cette patiente ressentait par moments la crainte obsédante de nourrir des pensées sexuelles à l'endroit de son fils aîné. On n'ignore point, par ailleurs, que certaines hystériques guérissent d'elles mêmes en donnant naissance à un enfant: dans mon expérience, cela arrive plus souvent lorsque l'enfant est un garçon plutôt qu'une fille.

Mentionnons ici un fait intéressant, se rattachant à la résolution de l'EEdipe chez des hommes présentant un symptôme d'impuissance. La puissance recouvrée est toujours associée à une capacité d'exhibition masculine qui faisait défaut à l'origine. Ce mécanisme n'a toutefois pas encore été suffisamment éclairé.

\section{La résolution du désir oedipien}

Je vais maintenant signaler à quels égards mes conceptions de la genèse et de la résolution du complexe d'Edipe diffèrent de celles de Freud. Dans un premier temps, je ne crois pas qu'il puisse y avoir une complète disparition des tendances œdipiennes. Même chez des individus normaux, on peut, en analyse, déceler le désir œdipien, à cette différence près qu'il n'est pratiquement plus pathogène, si bien qu'il trouve sa satisfaction dans des attitudes normales de la vie. Je ne crois pas en la validité de la théorie qui stipule que la disparition d'une tendance n'advient que de la voir éconduite ou déçue, sans l'aide d'autres facteurs. Comme je l'ai montré dans un autre texte, une tendance ne peut disparaître de la conscience qu'à deux conditions : elle peut être temporairement détruite en étant satisfaite, ou renvoyée dans l'inconscient lorsqu'intervient une tendance opposée. Aucune menace ni déception n'est assez

\footnotetext{
${ }^{13} \mathrm{Idem}$
} 
grande pour détruire une tendance, le seul effet étant alors de renvoyer à plus tard la réalisation de ce désir pressant, la force qui le motive demeurant intacte.

La tendance odipienne ne succombe ni aux menaces de castration, ni aux diktats du surmoi. La dimension de la castration est à l'origine un désir, à rapporter à l'envie d'être une femme. La menace ne devient effective que lorsque le conflit entre tendances masculines et féminines occupe le premier plan dans l'esprit de l'enfant, entraînant alors un refoulement de l'aspiration féminine. Le surmoi n'est en aucun cas un apport étranger à l'esprit de l'enfant, c'est cette partie de sa personnalité qui souhaite jouer le rôle du père. Le sentiment de culpabilité et la conscience punitive sont à renvoyer, en dernière instance, à l'opposition entre les parties masculine et féminine de la personnalité de l'enfant. Je ne partage donc pas l'avis de Freud quand il affirme que les tendances œdipiennes succombent finalement devant l'autorité du surmoi. C'est tout le contraire. Le surmoi doit s'acquérir, et la possibilité de castrer le père et d'en faire une femme est une condition nécessaire pour la résolution du désir œdipien. L'Edipe est résolu non point par la menace de castration mais par la faculté de castrer.

Freud doit reconnaître l'importance de l'éducation dans la résolution du conflit œdipien chez la petite fille, pour qui la menace de castration n'a pas d'effet, ce qui diminue en conséquence chez elle tout motif de formation du surmoi. Il n'y a pas à identifier, à mon sens, de mécanisme spécifique dans le cas de la fille. Ici aussi, une parfaite compréhension et intelligence $^{14}$ des trois sommets de l'alliance triangulaire est la clé de la résolution de la tendance œdipienne. J'ai déjà signalé que chez le patients indiens, le complexe de castration n'est jamais prédominant, bien que la menace de castration soit une mise en garde presque quotidienne dans les familles indiennes. Cela confirme ce que je soutiens ici : la menace de castration n'est pas la raison essentielle de la résolution de l'Édipe. Si une analyse superficielle de patients européens porte l'emphase sur l'importance du complexe de castration, lorsque l'analyse est approfondie, le désir d'être une femme se révèle en être l'élément central.

Traduction de Thamy Ayouch

\footnotetext{
${ }^{14}$ Idem
} 